\title{
Evaluación nasal en el abordaje quirúrgico de la patología selar
}

\author{
Nasal Evaluation in the Surgical Approach of Sellar Diseases
}

Dr. Homero Sariego $\mathrm{R}^{1}$, Dra. Mariana Bogado $\mathrm{C}^{2}$.

\begin{abstract}
RESUMEN
Introducción: $\theta$ abordaje quirúrgico de la hipófisis ha experimentado un importante desarrollo desde sus inicios; desde el abordaje transcraneal al transesfenoidal, siendo éste último el más utilizado para el tratamiento de la patología selar.

Objetivo: Comunicar la experiencia quirúrgica del manejo del septum y fosas nasales, en el abordaje selar en el Instituto de Neurocirugía Dr. Alfonso Asenjo (INCA).

Material y Método: Se realizó un estudio descriptivo-transversal de pacientes operados por vía transeptoesfenoidal en el INCA desde el 1ํ de enero de 2007 al 31 de marzo de 2008. Se expone la experiencia del abordaje y seguimiento de las complicaciones nasales.

Resultados: La edad promedio fue de 42,96 años, siendo 52,4\% de los casos mujeres y $47,6 \%$ hombres. Un $61 \%$ de los casos corresponde al primer abordaje, por 10 que casi un $40 \%$ de las cirugías se realiza en un tabique previamente intervenido. $\mathrm{La}$ mediana del retiro de tapones fue a los 4 días. No se utilizó antibióticos profilácticos.

Discusión y Conclusiones: $\mathrm{B}$ abordaje de Cottle es la técnica quirúrgica habitual. La reposición septal posterior nos ha permitido reoperar por esta vía, en períodos variables de tiempo, hasta cuatro veces sin dejar lesiones nasales. A pesar de no utilizarse antibióticos profilácticos, no se presentaron infecciones en esta serie.

Palabras claves: Abordaje septoesfenoidal, patología selar.
\end{abstract}

\begin{abstract}
Introduction: Since its beginnings, the surgical approach to the hypophysis has experienced an important development; from the transcranial to the transsphenoidal approach. The latter has evolved into the first line treatment method for sellar diseases.

Aim: To report the surgical experience in nasal septum management during sellar approach at the Instituto de Neurocirugía Dr. Alfonso Asenjo (INCA).

Material and Method: This study is a descriptive and transversal analysis of patients who underwent trans-septosphenoidal surgery at INCA, from January 1 15t, 2007
\end{abstract}

Médico de la Unidad de Otoneurología, Instituto de Neurocirugía e Investigaciones Cerebrales Alfonso Asenjo, Santiago.

2 Médico cirujano. Magíster en Salud Pública mención Epidemiología. Universidad de Chile. 
to March, 31st, 2008. The otorhinolaryngologic experience in the transsphenoidal approach is also presented.

Results: Average age of patients was 42.26 years, $52,4 \%$ of the cases were female and $47,6 \%$ male. $61 \%$ of the surgeries corresponded to a first intervention, which explains why nearly $40 \%$ of surgeries were performed in a septum that was previously manipulated. Nasal packing was removed within a median of 4 days. No prophylactic antibiotics were used.

Discussion and Conclusions: The current surgical technique used is the Cottle approach. Repositioning of the posterior septum allows surgical reinterventions using the same approach, even up to four times, in variable periods of time, without nasal lesions. No infections were reported in this study.

Key words: Trans-septosphenoidal approach, sellar pathology.

\section{INTRODUCCIÓN}

Históricamente el abordaje quirúrgico de la hipófisis ha experimentado un importante desarrollo desde su descripción inicial, evolución que no ha estado exenta de detractores y controversias. Durante el siglo XIX el abordaje de la patología selar era realizado por vía transcraneal; la vía frontal fue lograda con éxito por primera vez por Fedor Krause en Berlín, mientras que la vía subtemporal fue planteada por Victor Horsley en Londres, quien reportó una serie de 10 casos exitosos de abordaje selar transcraneal, tanto por vía frontal como subtemporal'1,2. Posteriormente numerosos neurocirujanos intentaron seguir esta tendencia, sin embargo, la alta morbimortalidad asociada a esta vía de abordaje hicieron imperioso el desarrollo de vías extracraneales para el abordaje de las patologías selares ${ }^{3,4}$. En 1907, Hermann Schloffer plantea el abordaje selar transesfenoidal, con la finalidad de mejorar los síntomas oftalmológicos presentados por los pacientes portadores de tumores hipofisiarios, siendo el primero en operar un paciente con un tumor de hipófisis por esta vía en forma exitosa con mejoría de la sintomatología visual ${ }^{5,6}$, para lo cual, utilizó una técnica quirúrgica modificada de la que se utilizaba en esa época para tratar la sinusitis etmoidal ${ }^{1}$. El acumulo de informaciones de experiencias al respecto, ha permitido el avance actual sobre el tema.

El abordaje transesfenoidal, es el resultado de un proceso evolutivo de más de 100 años, en el que los avances tecnológicos, el advenimiento de los corticoides perioperatorios, la antibioticoterapia y la radioscopía, la han transformado en la vía de abordaje más utilizada para el tratamiento de lesiones que comprometen la región selar-10, al igual que en casos seleccionados de lesiones paraselares y supraselares ${ }^{1}$.

En el Instituto de Neurocirugía Dr. Alfonso Asenjo (INCA) hasta el año 2000, el abordaje hipofisiario por vía nasal era realizado en la gran mayoría de los casos por los neurocirujanos tratantes, los cuales utilizaban la vía sublabial. Los casos realizados en conjunto con otorrinolaringólogos eran escasos. Al analizar la experiencia de los neurocirujanos destacaban algunos hechos: la dificultad para usar vasoconstrictores en el abordaje septal por riesgo de taquicardia e hipertensión intracraneana, el no uso de antibióticos en el posoperatorio y el retiro de tapones al segundo día posoperatorio. Estas indicaciones eran diametralmente opuestas a la formación otorrinolaringológica. Durante los últimos siete años todos los abordajes han sido realizados por otorrinolaringólogos.

La compleja evolución de los tumores selares operados, en particular, su recidiva, obliga al abordaje a través de un tabique ya intervenido, encontrando a veces sólo mucosa en la parte posterior, la que habitualmente queda destruida en la segunda intervención con las complicaciones por todos conocidas. Lo anterior, y teniendo la información y alguna experiencia en reparación de perforaciones septales, conduce a una posición más cuidadosa en el tratamiento del septum nasal considerando a 
cada paciente susceptible de un nuevo abordaje por la misma vía.

El objetivo de esta publicación es comunicar nuestra experiencia en relación con el manejo quirúrgico del septum y las fosas nasales en una casuística importante de nuestro medio. Esta cirugía se realiza en el contexto de los abordajes de patología selar en el INCA, donde también realizamos accesos endoscópicos los cuales no forman parte del objetivo, puesto que la agresión del septum y fosas nasales es mínimo y no extrapolable a las complicaciones clásicas de una septoplastía.

\section{MATERIAL Y MÉTODO}

Se realizó un estudio descriptivo de corte transversal de pacientes operados por vía transeptoesfenoidal en el INCA desde el $1^{0}$ de enero de 2007 al 31 de marzo de 2008. Para lo cual se revisaron las fichas clínicas de los pacientes operados en la institución durante el período previamente definido. La base de datos se creó a partir del software EpiData 3.02. La información se fue analizada con el programa estadístico SPSS 11.0. Se consideró un nivel de significación de $95 \%$.

El análisis descriptivo de los resultados obtenidos en este estudio se realizó utilizando cifras absolutas y porcentuales. Para las variables cuantitativas (edad, tiempo transcurrido entre abordajes, tiempo en el que se produce el retiro de tapones) se comprobó normalidad de la distribución de los datos mediante el Test de Shapiro-Wilk y homocedastacidad con el Test de Barlett. Para estas variables, se calculó medidas de tendencia central (promedio o mediana, según la distribución de los datos) y medidas de dispersión (desviación estándar). Para las variables cuantitativas discretas (número de reoperaciones), cualitativas (sexo, reposición de tabique y complicaciones) se calcularon medidas de distribución de frecuencia (cifras absolutas y porcentuales).

Se expondrá, además, la experiencia del abordaje quirúrgico otorrinolaringológico por vía transeptoesfenoidal en pacientes con tumores de hipófisis, para lo cual se utilizó el abordaje clásico de Cottle.

\section{Abordaje quirúrgico clásico de Cottle ${ }^{11}$}

Se realiza una incisión hemitransfixiante derecha en "C" invertida en el extremo ventrocaudal del tabique. Luego se realiza un túnel superior completo del lado izquierdo submucopercóndrico (túnel № 1). Posteriormente se realiza el túnel basal derecho, submucoperióstico (túnel № 2). El tercer túnel es el basal izquierdo, submucoperióstico (túnel № 3) El túnel 2 se une al túnel № 3.

Posteriormente se desinserta el tabique cartilaginoso del surco vomeriano, desviando el cartílago cuadrangular hacia derecha con la mucosa ipsilateral adherida, exponiendo la lámina perpendicular del etmoides. En caso de necesidad se realiza una alotomía.

Se unen los tres túneles, se desinserta la unión cartílago-lámina perpendicular y se desperiostiza ampliamente el tabique óseo bilateral hasta el esfenoides, siguiendo el relieve de la unión lámina perpendicular-vómer que en su extremo posterosuperior está en relación con los ostium esfenoidales. Con un cincel fino se secciona la lámina perpendicular desde la posición más alta permitida por la narina hacia los ostium. Con el mismo cincel se secciona el vómer hacia el piso del esfenoides. Con un cincel levemente curvo se secciona la parte posterior del tabique óseo muy próximo al rostrum. Se extrae el tabique óseo (Figura 1) quedando vómer inferior para evitar insuficiencia velopalatina.

\section{Cierre del abordaje}

Primero se debe detectar y reparar de las lesiones previas o producidas por el acto quirúrgico: Septo-desviaciones, rupturas de la mucosa, luxaciones a medial de los cornetes medios, etc. Luego se reposiciona el tabique óseo, total 0 fragmentado en empalizada apoyado sobre el periostio más intacto y se adosa la mucosa opuesta a él. Se introducen tapones de Merocel № 8 con tubos hasta las coanas para mantener la estabilización del tabique posterior mientras se repara el anterior y se cierra la incisión. Algunos pacientes con acromegalia tienen un crecimiento excesivo de la cara en particular de la nariz y son portadores insuficiencia respiratoria valvular, por 


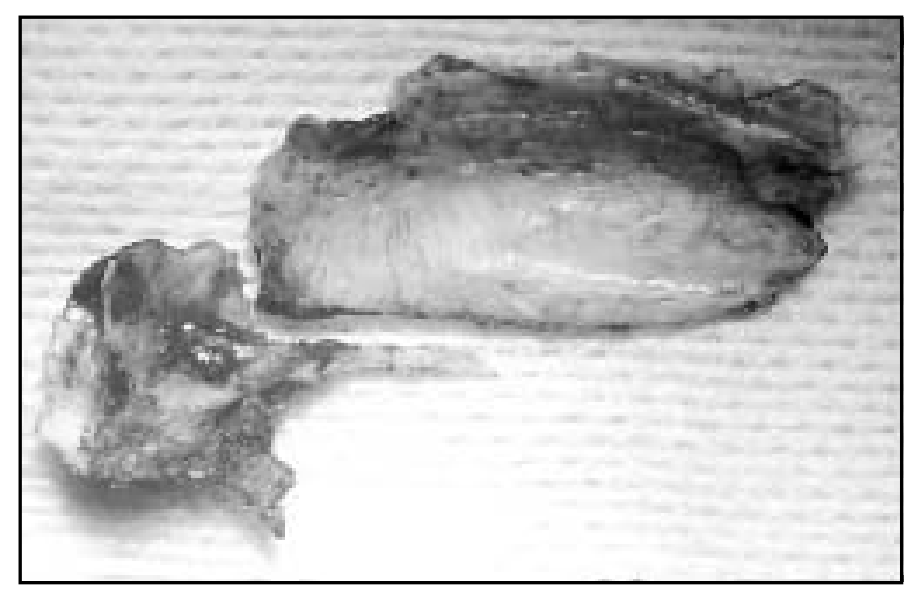

Figura 1. Rostrum Esfenoidal, Septum Posterior (Dr. Homero Sariego).

un ángulo nasal agudo. En ellos realizamos una corrección de la punta nasal.

Finalmente, terminado el trabajo septal anterior, se traccionan los tapones a nivel de las narinas.

\section{RESULTADOS}

Durante el período comprendido entre el $1^{0}$ de enero de 2007 y el 31 de marzo del 2008 se efectuaron 110 cirugías con abordaje transeptal, de los cuales 82 casos poseían la información requerida para ser incluidos en el estudio. La edad posee distribución normal ( $p=0,125$ en el test de Shapiro Wilk, $p=0,626$ en el test de Bartlett). El promedio de edad fue de $42,96 \pm 15,41$ años (rango: 10 a 75 años), la edad promedio por sexo fue de 44,23 $\pm 14,61$ años para los hombres y $41,82 \pm 16,20$ años para las mujeres. El $52,4 \%$ de los casos correspondió a sexo femenino y $47,6 \%$ a sexo masculino.

Al analizar el número de abordajes transeptales (Figura 2) se observó que el 61\% (número de casos $(n)=50)$ correspondió al primer abordaje; $28,1 \% \quad(n=23)$ a dos abordajes (una reintervención); $8,5 \%(n=7)$ a tres abordajes transeptales (dos reintervenciones) y $2,4 \%$

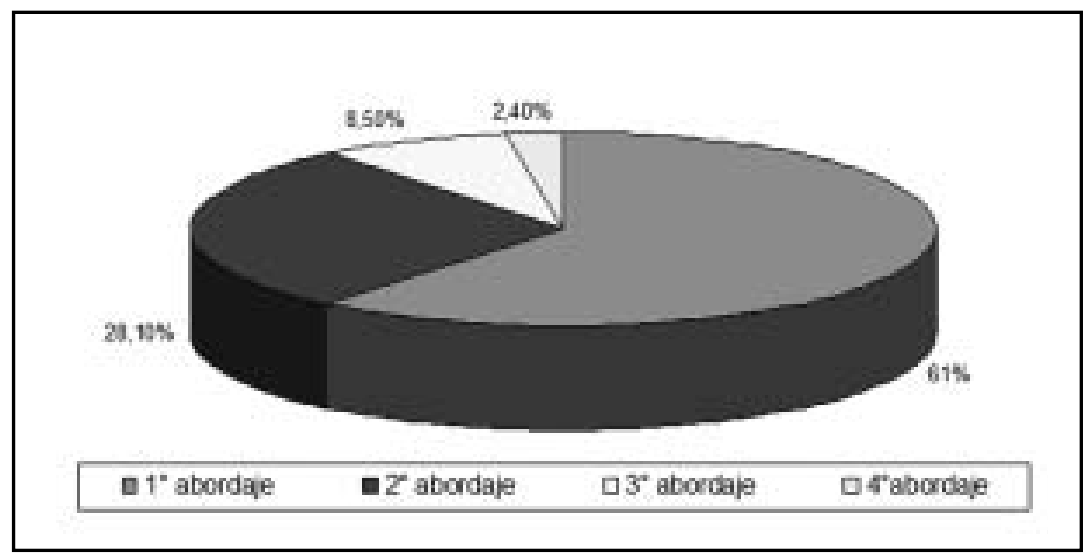

Figura 2. Número de abordajes transeptales. 
( $\mathrm{n}=2$ ) a un cuarto abordaje (tres reintervenciones) por vía transeptal.

Se realizó reposición del tabique en $69,5 \%(\mathrm{n}=$ $57)$ de los casos, mientras que en $30,5 \%(n=25)$ no fue posible reposicionarlo. La mediana del retiro de tapones fue a los 4 días.

La mediana del tiempo entre el primer y segundo abordaje fue de aproximadamente 3 años (1121 días) con un rango de 231 días a 27 años 6 meses. Entre el segundo y tercer abordaje la mediana fue de 5 años 6 meses (2057 días) con un rango desde 6 días (por complicación de fístula de líquido cefalorraquídeo (LCR)) hasta 16 años después del segundo abordaje. Los dos casos intervenidos por cuarta vez, fueron realizados a los 23 días y a los 2 meses 13 días del tercer abordaje.

Al analizar las complicaciones se describen 5 fístulas de $\operatorname{LCR}(6,1 \%)$, inherentes a la cirugía selar, inmediatas o dentro del posoperatorio pre$\mathrm{coz}$, reparadas quirúrgicamente y sin recidivas ni infecciones, una epistaxis masiva $(1,2 \%)$ y 2 episodios de sangrado al retirar los tapones $(2,4 \%)$.

\section{DISCUSIÓN}

La totalidad de los casos analizados corresponden a abordajes transeptales en los que se utilizó el abordaje clásico de Cottle.

Los tumores hipofisiarios pueden desarrollarse en todas las edades, el rango etario de nuestro estudio es similar al de otros estudios nacionales $^{8,12}$ pero menor que en un estudio internacional, en que el rango etario era de igual amplitud, pero 10 años mayor? ${ }^{7}$. La incidencia de los tumores hipofisiarios aumenta con la edad, describiéndose con mayor frecuencia sobre los 40 años ${ }^{7,8,12}$. El promedio de edad encontrado es similar a 10 descrito por otros estudios nacionales ${ }^{8}$, y levemente menor a lo descrito en Europa ${ }^{7}$. La distribución por sexo difiere de otros estudios nacionales, y de lo descrito en la literatura, en que se observa patología hipofisiaria con mayor frecuencia en el sexo femenino con una razón de 3:17,8,12, sin embargo, concuerda a con lo descrito en un estudio europe $0^{7}$ y los hallazgos descritos en autopsias, en que la distribución es igual en ambos sexos ${ }^{12}$.

Al analizar las complicaciones, al igual que en otros estudios la mayoría corresponde a neuroquirúrgicas. Las fístulas de LCR se describen en porcentajes similares en todos los estudios (entre $6 \%$ y $10 \%$ ). Mientras que los fenómenos hemorrágicos fueron menores que los descritos en la literatura ${ }^{8,12}$.

Se analizarán las indicaciones posoperatorias y complicaciones en el seguimiento posoperatorio naso-septal de los pacientes.

\section{Indicaciones posoperatorias}

En el período posoperatorio se indica reposo relativo, mantener el taponamiento nasal por 4 días promedio. Puede prolongarse por uno 0 dos días más en caso de fístulas de LCE en la silla turca. No se indica antibiótico en el período posoperatorio. Al cuarto día, al retirarse los tapones, se indica el alta. Eventualmente la hospitalización puede prolongarse para tratamiento del desbalance metabólico.

Se indica humidificación nasal con suero fisiológico y control a la semana, al mes o cuando lo requiera.

\section{Seguimiento}

Durante el seguimiento posoperatorio de los pacientes pueden presentarse las siguientes complicaciones:

Infecciones: Las cuales pueden ser precoces, al comprometer la incisión (ocasional). No se observa infección en alotomías, siendo en el período del análisis, esporádicas. No se encontró infecciones tardías, siendo posible la infección de los senos frontales, etmoidales o maxilares.

Epistaxis: Pueden ser precoces; en el período posoperatorio hospitalizado, en fosa nasal anterior que se ha cohibido con refuerzo del taponamiento, o tardías; generalmente posteriores que han requerido taponamiento anterior largo (ocasionales).

Disfunción ventilatoria: Es posible observar leves septodesviaciones no obstructivas. Ninguno ha sido reintervenido por causa otorrinolaringológica.

Trastornos del gusto y el olfato: Se observan hiposmias ocasionales.

Rinitis hiperreactivas: Algunas de tipo vasomotor.

Secreciones: Generalmente son costrosas en un comienzo, ceden con lavados. 


\section{CONCLUSIONES}

El promedio de edad de los pacientes intervenidos quirúrgicamente fue de 42,96 $\pm 15,41$ años, correspondiendo $52,4 \%$ de los casos a sexo femenino y $47,6 \%$ a sexo masculino. De los abordajes transeptales se observó que $61 \%$ de los casos corresponde al primer abordaje y que al menos un tercio de las cirugías se realiza con un tabique previamente intervenido, incluso hasta 27 años después. La mediana del retiro de tapones fue a los 4 días.

El abordaje maxila-premaxila de Cottle ${ }^{11}$, es habitual para los otorrinolaringólogos, excepto su proyección hacia el esfenoides. La exéresis parcial y cuidadosa del tabique óseo hasta el seno esfenoidal mismo y su reposición posterior, no tiene mayor complejidad quirúrgica.

La reposición septal posterior facilita las reoperaciones por esta vía, que en nuestra experiencia se realizó hasta cuatro veces en un mismo paciente, sin dejar lesiones nasales de consulta otorrinolaringológica. Cabe señalar que la resolución de la patología selar no depende de la técnica quirúrgica para acceder a ella sino de la excéresis total del tumor con su cápsula. Lograr este objetivo implica reoperaciones, fístulas de líquido cefalorraquídeo y su reparación inmediata. Lo anteriormente expuesto justifica dejar el septum nasal lo más anatómicamente posible.

En reoperaciones de varios años, sin reposición de tabique posterior consignado, fue posible encontrar un tabique óseo muy delgado, formado por el periostio, situación que no sucede con el pericondrio en la porción nasal anterior, por lo que se deben tratar de inmediato las perforaciones, con técnicas de rotación de colgajos bilaterales e interponiendo cartílagos o huesos, suturando el defecto con puntos de sutura reabsorbible dependiendo de la profundidad nasal 0 manteniéndolos apoyados por el taponamiento.

Muchas desviaciones anteriores son dependientes de una desviación del tabique posterior y amerita accionar sobre él para alinear el tabique en su conjunto

Finalmente, frente a la evidencia clínica de conductas terapéuticas posoperatorias neuroquirúrgicas, no se utiliza antibióticos profilácticos para prevenir posibles infecciones por tapones. Esto implica una revisión previa exhaustiva de la historia clínica, análisis radiológico nasosinusal y de la base de cráneo lo que es favorecido por la obligatoriedad de tomografía y/o resonancia magnética pre y posoperatoria y finalmente la vigilancia de los pacientes hospitalizados, en particular, inmunodeprimidos.

\section{REFERENCIAS}

1. Kanter AS, Dumont AS, Asthagiri ar, Oskouian RJ, Jane JA JR., Laws ER JR. The transsphenoidal approach. A historical perspective. Neurosurgical focus. 2005; 15; 18(4): e6.

2. TAN TC, BLACK P. Sir Victor Horsley (18571916): pioneer of neurological surgery. Neurosurgery 2002; 50: 607-11.

3. López 0, González J, Morales 0, Valdés N. Transeptosphenoidal surgery in prolactinsecreting hypophyseal adenomas. Rev Cubana Endocrinol 2005 [citado el 10 de marzo del 2008]; Disponible en: http://scielo.sld.cu/ scielo.php?script=sci_ arttext\&pid=S156129532005000200005\&lng=en\&nrm=iso\&tlng=es

4. Fatovi S, Gnsidi Z. Sellar Region Surgery in Croatia in the First Half of 20th Century. Croat Med J 2006; 47: 310-7.

5. LINDHOLM J. A century of pituitary surgery: Schloffer's legacy. Neurosurgery 2007; 61(4): 865-7; Discusión: 7-8.

6. Campero A, Cruz J, González S. El punto esfenoselar: Ubicación Anatómica y utilidad en 34 casos operados por vía Endonasal Transesfenoidal. Revista Argentina de Neurocirugía 2006; 20(7): 7-12.

7. Díaz A, Armengot M, Alba J, Barcia J, Basterra J. Abordajes Transnasales para los Tumores de Región Hipofisaria. Nuestra Experiencia. Acta Corrinolaringol Esp 2004; 54: 419-24.

8. Olavarría C, Stott C, Lemp M, Bustamante C, Schmidt N, Emerich M. Comparación de dos técnicas quirúrgicas para abordaje de la región selar: Transeptal transesfenoidal versus transnasal directa. Rev Otorrinolaringol Cir Cabeza Quello 2004; 64: 91-8. 
9. Kennedy DW, Cohn ES, Papel ID, Holliday MJ. Transsphenoidal approach to the sella: The Johns Hopkins experience. Laryngoscope 1984; 94: 1066-74.

10. Lanzino G, Laws ER JR (2001) Pioneers in the development of transsphenoidal surgery: Theodore Kocher, Oskar Hirsch, and Norman Dott. J Neurosurg 95(6): 1097-1103.

11. Cottle M. Concepts of nasal physiology as related to corrective nasal surgery. Arch Oolaryngol 1960; 72: 11-20.
12. Ortega E, Jaque R, Fernandez P, Muñoz A. Análisis de 50 casos de tumores hipofisiarios operados. Revista Chilena de Neurocirugía. 1995, Citado: 20 de junio, 2008. Disponible en línea en: http://www.neurocirugia.cl/revista/ revista_noviembre _2005/Trabajos\%20 Originales/(Microsoft\%20Word\%20-\%20ANALISIS\%20-DE\%2050\%20CASOS\%20DE\%20TUMORES\%2OHIPOFISIARIOS\%200PERADOS.pdf 